Research Paper

\title{
Systemic inflammation score predicts survival in patients with intrahepatic cholangiocarcinoma undergoing curative resection
}

\author{
Yong Zhang1,2,t, Shi-Ming Shi3,t, Hua Yang1,t, Liu-Xiao Yang2,t, Zheng Wang2, Xue-Dong Li², Dan Yin²,4, \\ Ying-Hong Shi², Ya Cao5 ${ }^{5}$ Zhi Dai ${ }^{2,4}$, Jian Zhou ${ }^{2,4,6, \bowtie}$, and Qing Chen ${ }^{1,} \bowtie$ \\ 1. Department of General Surgery, Zhongshan Hospital (South), Fudan University, Shanghai Public Health Clinical Center, Fudan University, Shanghai \\ 200083, China \\ 2. Liver Cancer Institute, Zhongshan Hospital, Fudan University, Key Laboratory of Carcinogenesis and Cancer Invasion, Ministry of Education, Shanghai \\ 200032, China \\ 3. Department of Radiation Oncology, Zhongshan Hospital, Fudan University, Shanghai 200032, China \\ 4. Institute of Biomedical Sciences, Fudan University, Shanghai 200032, China \\ 5. Cancer Research Institute, Central South University; Key Laboratory of Carcinogenesis and Cancer Invasion, Ministry of Education, Changsha 410078, \\ China \\ 6. State key laboratory of genetic engineering, Fudan University, Shanghai 200032, China.
}

†These authors contributed equally to this work.

$\triangle$ Corresponding authors: Qing Chen, Department of General Surgery, Zhongshan Hospital (South), Fudan University; Shanghai Public Health Clinical Center, Fudan University, 921 Tongxin Road, Shanghai 200083, China. Phone: +86-21-37990333, E-mail: chenqing 84@163.com; or Jian Zhou, Liver Cancer Institute, Zhongshan Hospital, Fudan University, 136 Yi Xue Yuan Road, Shanghai 200032, China. Phone: +86-21-64041990; Fax: +86-21-64037181, E-mail: zhou.jian@zs-hospital.sh.cn.

(c) Ivyspring International Publisher. This is an open access article distributed under the terms of the Creative Commons Attribution (CC BY-NC) license (https://creativecommons.org/licenses/by-nc/4.0/). See http://ivyspring.com/terms for full terms and conditions.

Received: 2018.04.25; Accepted: 2018.11.07; Published: 2019.01.01

\begin{abstract}
Inflammation has a critical role in the development and progression of cancers. We developed a novel systemic inflammation score (SIS) based on lymphocyte, monocyte, and CA19-9 and explored its prognostic value in intrahepatic cholangiocarcinoma (ICC). From January 2005 to December 2011, 322 consecutive ICC patients who underwent curative resection in our center were included in this study, and validated in a retrospective study of 126 patients enrolled from 2012 to 2014. Clinicopathological variables including preoperative serum CA19-9 and LMR were analyzed. The cutoff values of CA19-9 and LMR were determined based on receiver operating characteristics curve analysis in the primary cohort. Kaplan-Meier curves and multivariate Cox-regression analyses were calculated for time to recurrence (TTR) and overall survival (OS). In univariate analysis of all patients, all three inflammatory and tumor marker including NLR $\geq 2.49(P<0.001)$, LMR $\leq 4.45$ $(P=0.002)$, and CA19-9 $\geq 89(P<0.001)$ were associated with poor prognoses. When omitting SIS in multivariate analysis, preoperative LMR $(P=0.006)$ and serum CA19-9 $(P<0.001)$ were independent predictors of OS. In addition, elevated CA19-9 $(P=0.001)$, multiple tumors $(P<0.001)$, and lymph node metastasis $(P<0.001)$ were significant predictors of worse recurrence free survival. Moreover, high SIS was significantly associated with aggressive tumor behaviours including large tumor size $(P<0.001)$, multiple tumors $(P=0.033)$, lymphonodus node metastasis $(P=0.001)$, and high TNM stage $(P<0.0001)$. Finally, univariate and multivariate analyses revealed the SIS was an independent predictor for TTR $(\mathrm{HR}=2.077,95 \% \mathrm{Cl}, 1.365-3.162, P=0.001)$ and OS $(\mathrm{HR}=3.13395 \% \mathrm{Cl}, 2.058-4.769, P<0.001)$. These results were further confirmed in the validation cohort. In conclusions, our findings demonstrate that the SIS as a potentially powerful prognostic biomarker in ICC that predicts poor clinical outcomes and is a promising tool for ICC treatment strategy decisions.
\end{abstract}

Key words: lymphocyte-monocyte ratio, CA19-9, systemic inflammation, intrahepatic cholangiocarcinoma, liver resection, prognosis

\section{Introduction}

Intrahepatic cholangiocarcinoma (ICC), originating from the intrahepatic biliary tree, is the second most common primary hepatic malignancy

tumor. It accounts for $5 \%$ to $30 \%$ of all primary liver cancers $[1,2]$. The incidence and mortality of ICC has been rapidly increasing in the United States [3, 4]. ICC 
is one of the most fatal cancers, surgical resection remains the mainstay of curative intent treatment for ICC $[5,6]$. Unfortunately, prognosis after partial hepatic resection is unsatisfactory, with 5-year survival rates of $30 \%$ to $40 \%$ after hepatectomy and ICC patients with unresectable disease have a median survival of only 12-15 months [7-9]. Owing to treatment-refractory disease with dismal outcome, the early detection of ICC and identification prognostic and protective factors are beneficial for improving ICC patients' outcome.

In the last decade, cancer-related inflammation has been verified to be involved in cancer development and progression, and in particular facilitates tumor cell proliferation, survival, angiogenesis, and metastasis [10, 11]. Most ICC patients have a background of hepatitis B virus and/or hepatitis C virus infection, choledocholithiasis, and primary sclerosing cholangitis [12-14], suggesting a potential link between chronic inflammation and ICC development. Therefore, studies on the mechanisms of cancer-related inflammation and tumor progression in ICC remain to be extensively investigated.

Cancer-related inflammation has emerged as the seventh hallmark of cancers, and the key determinant effects of tumor-associated innate immune cells have been suggested in a numerous cancers $[15,16]$. There is recent evidence that systemic inflammatory response, such as neutrophil-lymphocyte ratio (NLR), lymphocyte-monocyte ratio (LMR), modified Glasgow prognostic score (mGPS), prognostic nutritional index (PNI), and C-reactive protein (CRP) had been proposed and investigated as prognostic biomarkers in patients with several solid tumors [17-24]. In this study, we created a novel prognostic score named systemic inflammation score (SIS) basing on preoperative serum CA19-9 and LMR, which were both verified to be independently associated with ICC patients' outcomes. Furthermore, the prognostic utility of the SIS in ICC patients who underwent curative resection was evaluated in two independent cohorts. We found that the SIS was a promising independent predictive factor for prognosis of patients with ICC after curative surgical resection and that the increased recurrence rate in patients with high SIS score.

\section{Materials and Methods}

\section{Patients and follow up}

This retrospective study included 322 consecutive patients with ICC (the primary cohort, $n=322$ ), who underwent curative intent hepatic resection at our institution (Liver Surgery Department of Zhongshan Hospital, Fudan University, Shanghai,
China) between January 2005 and December 2011. From 2012 to 2014, a validation cohort of patients with ICC (the validation cohort, $n=126$ ) undergoing resection was recruited. The eligibility criteria were as follows: complete resection of macroscopic liver tumors and histopathological diagnosis of ICC, no history of previous anti-cancer therapies and other malignancies, availability of data on all clinical and laboratory features and treatments given, as well as outcome, and follow-up. Patients who underwent preoperative therapies (e.g., transcatheter arterial chemoembolization, percutaneous ethanol injection, or, radiofrequency ablation) were excluded from this study. Part of the physical examinations, serum tumor markers included CA19-9, and a-fetoprotein (AFP), routine blood and liver function tests were performed within one week prior to surgery. The Research Ethics Committee of Zhongshan Hospital approved this study, and written informed consent was obtained from all patients. The study was censored on August 1, 2018.

\section{Follow-up Strategy and Recurrence Pattern}

The patients follow-up and postoperative treatment were administrated as described previously according to our established guidelines [25, 26]. Briefly, after discharge, all patients were followed up once every two months for the first two years after hepatectomy and thereafter every three to six months interval or until death. At each of the follow-up visits, peripheral blood was routinely taken for routine blood, liver function tests and serum tumor markers including CA19-9, and AFP, and an abdominal ultrasound was performed. Contrast-enhanced CT or MRI was performed once every six months or earlier if tumor recurrence or metastasis was suspected. Further investigation was carried out when clinically indicated, such as positron emission tomography CT (PET-CT). While ICC recurrence was being confirmed, a second hepatectomy, radiofrequency ablation, percutaneous ethanol injection, transcatheter arterial chemoembolization, or external radiotherapy were administered according to the number, size, and site of the recurrent tumors [27]. Overall survival (OS) and time to recurrence (TTR) were used as primary end points. OS was calculated from the dates of operation to the dates of death or the dates of last follow-up. TTR was calculated from the interval between the dates of operation and the first recurrence or from the dates of operation to the dates of last follow-up (for the patients without recurrence).

\section{Statistical Analysis}

Statistical analyses were assessed using SPSS 19.0 software (SPSS, Chicago, IL, USA). Data were presented as the means \pm standard deviation. The 
optimal cutoff values of pretreatment LMR and CA19-9 were evaluated using receiver operating curve (ROC) analysis. Categorical data were analyzed by the $x^{2}$ test or Fisher's exact test. Kaplan-Meier survival curves were used for calculating the OS and TTR. Univariate and multivariate analyses were performed using the Cox proportional hazards regression model. Differences were considered statistically significant at $P<0.05$.

\section{Results}

\section{Patient Characteristics}

The clinicopathologic features of ICC patients were presented in Table 1 . In the training cohort, the mean age of the patients was 57.9 years (range, 27.0-81.0 years); the hepatitis etiology was the hepatitis B virus in $38.2 \%$ of the patients and the hepatitis $C$ virus in $0.62 \%$ the patients; and $28.6 \%$ of the patients exhibited either macrovascular invasion $(14.3 \%)$ or lymphonodus node metastasis $(17.4 \%)$. The median follow-up time was 44.0 months (range, 2.7-100.5 months), 187 (58.1\%) patients had died and $191(59.3 \%)$ patients were confirmed with tumor recurrence at last follow-up. The postoperative 1-, 3-, and 5-year survival rates were $75.0 \%, 47.8 \%$, and $35.2 \%$, respectively. The postoperative $1-, 3-$, and 5-year recurrence free survival rates were $56.6 \%, 39.0$ $\%$, and $32.3 \%$, respectively (Fig. 1 ).

In the validation cohort $(n=126)$, the median follow-up time was 38.3 months (range, 1.0 to 80 months). The median TTR was 18 months (range, 2 to 76 months), and the postoperative 1- and 3-year recurrence rates were $43.1 \%$ and $63.1 \%$, respectively. The median OS time was 29.3 months (range, 3.5 to 80 months), and the 1-and 3-year OS rates were $72.8 \%$ and $45.3 \%$, respectively. The clinicopathologic characteristics were similar between the two cohorts, with the exception of HBsAg and AFP. The primary cohort included more HBsAg and AFP than those in the validation cohort (Supplementary Table 1).

\section{The Optimal Cut-Offs of LMR and CA19-9}

Next, a LMR of 4.45 was the optimal cutoff point for postoperative prognosis, using time-dependent receiver operating characteristic curve. Therefore, all the patients were stratified into two groups: a low $(<4.45)$ LMR group $(n=210)$ and a high $(\geq 4.45)$ LMR group $(n=112)$. Similarly, the optimal cutpoint of 89 $\mathrm{U} / \mathrm{ml}$ was identified for the CA19-9. High and low groups were created as for the LMR using the same method.

Table 1. Correlation between the factors and clinicopathologic characteristics in ICC (Cohort 1, $n=322$ )

\begin{tabular}{|c|c|c|c|c|c|c|c|c|c|c|c|}
\hline \multirow[t]{2}{*}{ Clinicopathological Indexes } & & \multirow{2}{*}{\multicolumn{2}{|c|}{ LMR }} & \multirow{2}{*}{$P$} & \multirow{2}{*}{\multicolumn{2}{|c|}{ CA19-9 }} & \multirow{2}{*}{$P$} & \multirow{2}{*}{\multicolumn{3}{|c|}{ SIS }} & \multirow{2}{*}{$P$} \\
\hline & & & & & & & & & & & \\
\hline \multirow[t]{2}{*}{ Age(year) } & $\leq 50$ & 23 & 62 & 0.000 & 57 & 28 & 0.782 & 23 & 40 & 22 & 0.953 \\
\hline & $>50$ & 187 & 50 & & 155 & 82 & & 61 & 116 & 60 & \\
\hline \multirow[t]{2}{*}{ Sex } & female & 76 & 52 & 0.074 & 74 & 54 & 0.138 & 39 & 58 & 31 & 0.346 \\
\hline & male & 134 & 60 & & 128 & 66 & & 45 & 98 & 51 & \\
\hline \multirow[t]{2}{*}{ HBsAg } & negative & 135 & 64 & 0.21 & 125 & 74 & 0.146 & 49 & 88 & 62 & 0.011 \\
\hline & positive & 75 & 48 & & 87 & 36 & & 35 & 68 & 20 & \\
\hline \multirow[t]{2}{*}{$\mathrm{HCV}$} & negative & 209 & 111 & $1^{*}$ & 211 & 109 & $1^{*}$ & 84 & 155 & 81 & $0.746^{*}$ \\
\hline & positive & 1 & 1 & & 1 & 1 & & 0 & 1 & 1 & \\
\hline \multirow[t]{2}{*}{$\operatorname{AFP}(\mathrm{ng} / \mathrm{ml})$} & $\leq 20$ & 186 & 100 & 0.846 & 188 & 98 & 0.911 & 84 & 128 & 74 & $0.000^{*}$ \\
\hline & $>20$ & 24 & 12 & & 24 & 12 & & 0 & 28 & 8 & \\
\hline \multirow[t]{2}{*}{ Child-Pugh } & A & 202 & 109 & $0.753^{*}$ & 208 & 103 & $0.05^{\star}$ & 83 & 151 & 77 & $0.183^{*}$ \\
\hline & B or C & 8 & 3 & & 4 & 7 & & 1 & 5 & 5 & \\
\hline \multirow[t]{2}{*}{ Liver cirrhosis } & no & 156 & 80 & 0.581 & 149 & 87 & 0.090 & 58 & 113 & 65 & 0.317 \\
\hline & yes & 54 & 32 & & 63 & 23 & & 26 & 43 & 17 & \\
\hline \multirow[t]{2}{*}{ Tumor size $(\mathrm{cm})$} & $\leq 5$ & 90 & 55 & 0.283 & 115 & 30 & 0.000 & 46 & 77 & 22 & 0.000 \\
\hline & $>5$ & 120 & 57 & & 97 & 80 & & 38 & 79 & 60 & \\
\hline \multirow[t]{2}{*}{ Tumor number } & single & 163 & 81 & 0.291 & 164 & 80 & 0.358 & 72 & 110 & 62 & 0.033 \\
\hline & multiple & 47 & 31 & & 48 & 30 & & 12 & 46 & 20 & \\
\hline \multirow[t]{2}{*}{ Lymphonodus node metastasis } & yes & 41 & 15 & 0.167 & 25 & 31 & 0.000 & 5 & 29 & 22 & $0.001^{*}$ \\
\hline & no & 169 & 97 & & 187 & 79 & & 79 & 127 & 60 & \\
\hline \multirow[t]{2}{*}{ Microvascular invasion } & yes & 33 & 13 & 0.316 & 29 & 17 & 0.666 & 9 & 24 & 13 & 0.562 \\
\hline & no & 177 & 99 & & 183 & 93 & & 75 & 132 & 69 & \\
\hline \multirow[t]{3}{*}{ Tumor differentiationa } & poor & 46 & 22 & 0.903 & 49 & 19 & 0.207 & 17 & 37 & 14 & 0.249 \\
\hline & moderated & 130 & 71 & & 133 & 68 & & 52 & 100 & 49 & \\
\hline & well & 34 & 19 & & 30 & 23 & & 15 & 19 & 19 & \\
\hline \multirow[t]{2}{*}{ TNM stageb } & $\mathrm{I}+\mathrm{II}$ & 155 & 93 & 0.061 & 178 & 70 & 0.000 & 75 & 121 & 52 & 0.000 \\
\hline & III+IVA & 55 & 19 & & 34 & 40 & & 9 & 35 & 30 & \\
\hline
\end{tabular}

Boldface type indicates significant values.

Abbreviations: AFP, alpha-fetoprotein; CA19-9, carbohydrate antigen 19-9; HBsAg, hepatitis B surface antigen; HCV, hepatitis C virus; LMR, lymphocyte-to-monocyte ratio; TNM, tumor-node-metastasis; SIS, combination of preoperative serum CA19-9 and LMR. I, CA19-9Low/LMR High; II, CA19-9 High /LMRHigh and CA19-9 Low/LMR Low; III, CA19-9 High/LMR Low.

a Tumor differentiation was determined according to the "British Society of Gastroenterology guidelines on the management of cholangiocarcinoma".

b TNM stage: American Joint Committee on Cancer 7th edition staging for intrahepatic cholangiocarcinoma. *Fisher's exact tests; chi-square tests for all other analyses. 

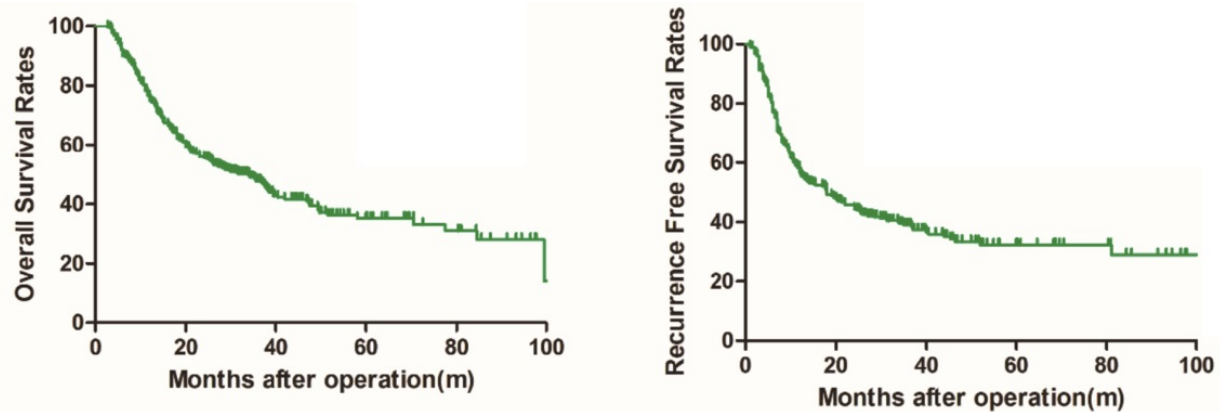

\begin{tabular}{|l|c|c|c|c|c|c|c|c|c|c|c|c|c|}
\hline \multicolumn{1}{|c|}{ OS } & \multicolumn{5}{|c|}{ NO. at Risk (\% survival) } & \multicolumn{5}{c|}{ RFS } & \multicolumn{5}{|c|}{ NO. at Risk (\% survival) } \\
\hline Year & 0 & 1 & 2 & 3 & 4 & 5 & & 0 & 1 & 2 & 3 & 4 & 5 \\
\hline All & 322 & 243 & 175 & 110 & 52 & 33 & & 322 & 171 & 124 & 75 & 34 & 20 \\
patients & & $(75.0)$ & $(55.4)$ & $(47.8)$ & $(39.5)$ & $(35.2)$ & & & $(56.6$ & $(44.4)$ & $(39.0)$ & $(33.4)$ & $(32.3)$ \\
\hline
\end{tabular}

Figure 1. Kaplan-Meier curve showing overall survival and recurrence-free survival of patient undergoing curative resection for ICC (Cohort 1 , $n=322$ ).

For the CA19-9, $212(65.8 \%)$ patients were in the low group, whereas $110(34.2 \%)$ were in the high group. In addition, the discrimination ability of inflammatory and tumor markers were compared by the area under the ROC curve (AUC) for OS. The AUCs for LMR and CA19-9 were 0.57 (95\% CI, 0.51-0.64, $P=0.034)$, and 0.63 (95\% CI, 0.57-0.69, $P=0.0001$ ), respectively (Supplementary Fig. 1).

\section{Associations of LMR, CA19-9, and SIS with clinicopathological parameters}

The relationships between the LMR, CA19-9, and SIS and clinicopathologic parameters are summarized in Table 1. We found that a low LMR was associated with older age $(P<0.001$, Table 1$)$. We noted that patients with a low LMR were likely to be older than 50 years $(89.0 \%)$ compared to those with high LMR $(44.6 \%)$. Scatter diagram was used to investigate the correlation between perioperative LMR and NLR. Scatter-plot analyses showed a significant negative linear correlation between perioperative LMR and NLR $(n=322, r=-0.3870, P<0.0001$; Supplementary Fig. 2). Specifically, high NLR was more likely in the low LMR group $(72.4 \%)$ than in the high LMR group (22.3\%). In addition, CA19-9 $\geq 89 \mathrm{U} / \mathrm{ml}$ was associated with high Child-Pugh scores $(P=0.05)$, large tumor size $(P<0.001)$, lymphonodus node metastasis $(P<0.001)$, and high TNM stage $(P<0.001$, Table 1$)$. An elevated SIS score was associated with HBsAg positive $(P=0.011)$, high AFP level $(P<0.001)$, large tumor size $(P<0.001)$, and multiple tumors $(P=0.033)$, lymphonodus node metastasis $(P=0.001)$, and high TNM stage $(P<0.0001$, Table 1$)$.

\section{The prognostic significance of SIS in ICC patients in the training cohort}

The Kaplan-Meier curves indicated that the high LMR was significantly associated with prolonged OS
( $P=0.0017$, Fig. 2A) and RFS ( $P=0.0453$, Fig. 2D). In addition, our results indicated that the $1-, 3-$, and 5-OS rates in the LMR ${ }^{\text {High }}$ ICC patients were significantly increased than the survival rates in the LMR ${ }^{\text {Low }}$ patients $(82.0 \%$ versus $71.3 \%, 62.2 \%$ versus $41.1 \%$, and $43.7 \%$ versus $31.0 \%$, respectively; $P=0.0017$, Fig. 2A). Similarly, LMR Low ICC patients had the worse prognosis at 1-, 3-, and 5-years, with worse RFS rates than $\mathrm{LMR}^{\mathrm{High}}$ patients $(53.1$ versus $62.8 \%, 35.8 \%$ versus $44.7 \%$, and $27.8 \%$ versus $40.3 \%$, respectively; $P=0.0453$, Fig. 2D).

The Kaplan-Meier curves revealed that the high CA19-9, and SIS score were both significantly associated with shorter OS $(P<0.0001$, and $P<0.0001$, respectively; Fig.2B and $C)$ and RFS $(P<0.0001$, and $P<0.0001$, respectively; Fig. $2 \mathrm{E}$ and F). Our results revealed that for the low CA19-9 group, the 1-, 3-, and 5 -year OS rates were $85.8 \%, 58.1 \%$, and $44.2 \%$, respectively. These results were significantly better compared with the high CA19-9 group who had rates of $54.3 \%, 27.8 \%$, and $18.1 \%$ for $1-, 3-$, and 5 -year OS, respectively $(P<0.0001$, Fig.2B). The results for RFS rates for ICC patients in the low and high CA19-9 groups are shown in Fig. 4E. The 1-, 3-, and 5-year RFS rates were significantly lower in the high CA19-9 group $(36.1 \%, 23.9 \%$, and $20.5 \%$, respectively) compared with the low CA19-9 group (66.6\%, 46.2\%, and $37.8 \%$, respectively; $P<0.0001$, Fig. 2E). Thus, these data indicated that ICC patients after hepatic resection with high CA19-9 $\geq 89 \mathrm{U} / \mathrm{ml}$ should be closely monitored for ICC recurrence.

When evaluating the combined effect of preoperative serum CA19-9 and LMR on ICC prognosis, our results demonstrated that the 1-, 3-, and 5-year OS rates for CA19-9 High/LMR Low patients were $48.8 \%, 23.0 \%$, and $15.6 \%$, respectively, and were significantly lower than the OS rates in the 
CA19-9Low/LMR ${ }^{\text {High }}$ patients $(85.7 \%, 65.7 \%$, and $48.9 \%$, respectively; Fig. 2C). The 1-, 3-, and 5-year RFS rates in the CA19-9Low/LMR ${ }^{\text {High }}$ patients were $67.6 \%, 50.9 \%$, and $45.3 \%$, respectively, which were significantly higher than those in the CA19-9 High/LMR Low patients $(30.7 \%, 24.8 \%$, and $19.9 \%$, respectively; Fig. 2F).

Results from our univariate analysis indicated that tumor size, tumor number, lymphonodus node metastasis, TNM stage, NLR , LMR, CA19-9, and SIS were prognostic factors of OS and TTR, multivariate analyses revealed that SIS were independent prognostic factors for both OS (HR=3.133 95\% CI, 2.058-4.769, $P<0.001$; Table 2) and TTR (HR=2.077, 95\% CI, 1.365-3.162, $P=0.001$; Table 2), together with multiple tumors and tumor-node metastasis (Table 2). Whereas age, gender, hepatitis history, AFP, Child-Pugh score, liver cirrhosis, and tumor differentiation had no prognostic significance for OS and TTR (Table 2). When omitting SIS in multivariate analysis, preoperative serum CA19-9 and LMR were both verified to be independently associated with ICC patients' prognosis (Table 3). Elevated LMR was associated with better OS $(\mathrm{HR}=0.636,95 \% \mathrm{CI}$ : $0.461-0.878, P=0.006$, Table 3$)$. While, multiple tumors, lymphonodus node metastasis, high TNM stage, elevated NLR and CA19-9 were independent predictors of worse OS (Table 3). Meanwhile, patients with elevated CA19-9, multiple tumors, and lymphonodus node metastasis were significant predictors of worse recurrence free survival (Table 3 ). In addition, the discrimination ability of the SIS, as assessed by AUC, was $0.652(0.593-0.712, P<0.001)$ for OS (Fig. 3), which was higher than other clinical indexes (CA19-9, LMR, NLR, and PLR) (Fig. 3). Previous study shown that PNI had been identified as independent prognostic factors in ICC advance patients [17]. However, we found that the PNI was not a promising independent predictive factor for prognosis of patients with ICC after surgery (Supplementary Fig. 3).

\section{Validation of the SIS in an independent cohort}

In the present study, the prognostic utilities of the preoperative LMR, serum CA19-9, and SIS were further confirmed in an independent validation cohort of patients with ICC (the validation cohort, $n=126$ ). The results were similar to those obtained from the training cohort (Fig. 2). The high LMR also significantly correlated with prolonged OS $(P=0.0064$; Fig. 4A) and RFS ( $P=0.0122$; Fig. $4 \mathrm{D})$. The high serum CA19-9 and SIS remained associated with shorter OS $(P<0.0001$ and $P<0.01$, respectively; Fig. $4 \mathrm{~B}$ and $4 \mathrm{C})$ and RFS $(P<0.0001$ and $P<0.01$, respectively; Fig. $4 \mathrm{E}$ and $4 \mathrm{~F})$. Univariate and multivariate analyses demonstrated that the high SIS was significantly correlated with shorter TTR (HR, 2.083; 95\% CI, 1.091-3.976; $P=0.026$; HR, 3.946; 95\% CI, 1.913-8.138, respectively; $P<0.0001)$ and $\mathrm{OS}(\mathrm{HR}, 3.213 ; 95 \% \mathrm{CI}$, 1.365-7.565; $P=0.008$; HR, 9.666; 95\% CI, 3.411-27.394; $P<0.0001$, respectively; Supplementary Table 2).
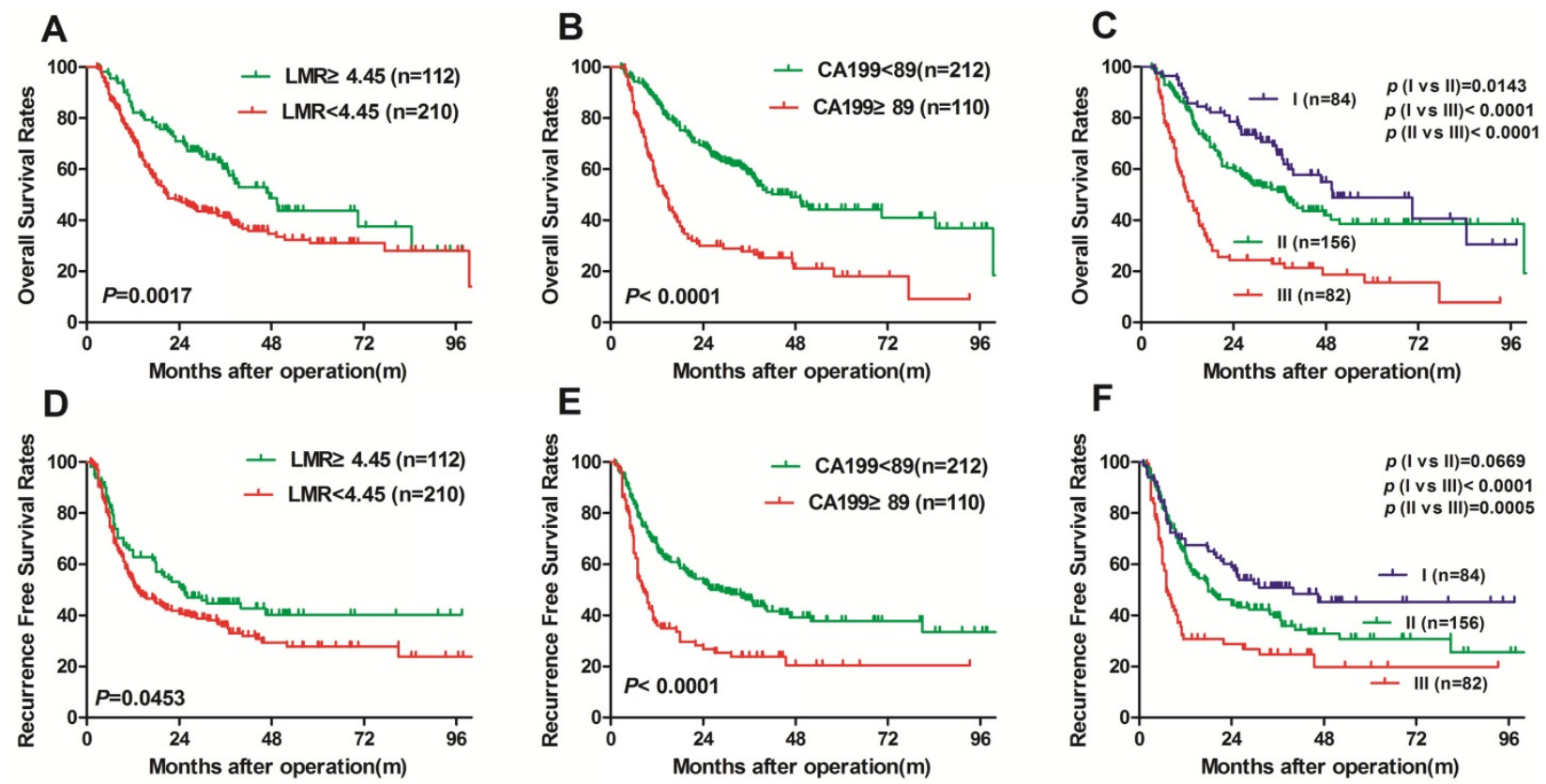

Figure 2. Prognostic values of preoperative LMR and serum CA19-9 using Kaplan-Meier analysis in the training cohort (Cohort 1, $n=322$ ). Kaplan-Meier analysis for overall survival according to (A) preoperative LMR, (B) preoperative serum CA 19-9, (C) combination of preoperative LMR and serum CA19-9. Kaplan-Meier analysis for recurrence-free survival according to (D) preoperative LMR, (E) preoperative serum CA19-9, (F) combination of preoperative LMR and serum CA19-9. SIS, combination of preoperative LMR and serum CA19-9. I, CA19-9Low/LMRHigh; II, CA19-9 High/LMRHigh and CA19-9 Low/LMR Low; III, CA19-9 High/LMR Low. 
Table 2. Univariate and Multivariate analyses of prognostic factors in ICC (Cohort $1, n=322)$

\begin{tabular}{|c|c|c|c|c|}
\hline \multirow[t]{2}{*}{ Variable } & \multicolumn{2}{|l|}{ TTR } & \multicolumn{2}{|l|}{ OS } \\
\hline & HR $(95 \% \mathrm{CI})$ & $P$ & HR $(95 \%$ CI $)$ & $P$ \\
\hline \multicolumn{5}{|l|}{ Univariate analysis } \\
\hline Age, year ( $\leq 50$ vs. $>50)$ & $1.160(0.841-1.598)$ & 0.366 & $1.079(0.780-1.493)$ & 0.647 \\
\hline Sex (female vs. male) & $1.063(0.794-1.422)$ & 0.683 & $1.143(0.850-1.538)$ & 0.376 \\
\hline HBsAg (negative vs. positive) & $0.660(0.271-1.171)$ & 0.360 & $1.090(0.505-2.352)$ & 0.826 \\
\hline HCV (negative vs. positive) & $0.645(0.206-2.018)$ & 0.451 & $1.333(0.330-5.380)$ & 0.686 \\
\hline $\mathrm{AFP}, \mathrm{ng} / \mathrm{ml}(\leq 20$ vs. $>20)$ & $0.897(0.558-1.441)$ & 0.652 & $0.897(0.558-1.442)$ & 0.654 \\
\hline Child-Pugh (A vs. B or C) & $0.660(0.271-1.605)$ & 0.360 & $1.090(0.505-2.352)$ & 0.826 \\
\hline Liver cirrhosis (no vs. yes) & $1.254(0.915-1.719)$ & 0.159 & $1.187(0.863-1.633)$ & 0.292 \\
\hline Tumor size, $\mathrm{cm}(\leq 5$ vs. $>5)$ & $1.349(1.012-1.798)$ & 0.042 & $1.486(1.107-1.994)$ & 0.008 \\
\hline Tumor number (single vs. multiple) & $1.839(1.345-2.515)$ & 0.000 & $1.636(1.191-2.248)$ & 0.002 \\
\hline Lymphonodus node metastasis (no vs. yes) & $2.532(1.792-3.577)$ & 0.000 & $2.895(2.066-4.058)$ & 0.000 \\
\hline Microvascular invasion (no vs. yes) & $1.545(1.066-2.240)$ & 0.022 & $1.272(0.856-1.892)$ & 0.234 \\
\hline Tumor differentiationa (P vs. M,W) & $1.304(0.976-1.740)$ & 0.072 & $1.126(0.839-1.511)$ & 0.428 \\
\hline TNM stage $^{\mathrm{b}}(\mathrm{I}+\mathrm{II}$ vs. III+IVA) & $2.042(1.483-2.813)$ & 0.000 & $2.459(1.798-3.364)$ & 0.000 \\
\hline NLR (low vs. high) & $1.426(1.069-1.902)$ & 0.016 & $1.782(1.322-2.402)$ & 0.000 \\
\hline LMR (low vs. high) & $0.735(0.542-0.997)$ & 0.048 & $0.604(0.439-0.831)$ & 0.002 \\
\hline CA19-9, U/ml ( $\leq 89$ vs. $>89)$ & $1.960(1.460-2.632)$ & 0.000 & $2.536(1.894-3.395)$ & 0.000 \\
\hline \multicolumn{5}{|l|}{ SIS } \\
\hline I & 1 (Referent) & & 1 (Referent) & \\
\hline II & $1.369(0.950-1.973)$ & 0.092 & $1.442(0.975-2.133)$ & 0.067 \\
\hline III & $2.429(1.621-3.640)$ & 0.000 & $3.577(2.378-5.382)$ & 0.000 \\
\hline \multicolumn{5}{|l|}{ Multivariate analysis ${ }^{1}$} \\
\hline Tumor size, $\mathrm{cm}(\leq 5 \mathrm{vs.}>5)$ & $1.030(0.743-1.426)$ & 0.860 & $1.014(0.733-1.402)$ & 0.934 \\
\hline Tumor number (single vs. multiple) & $1.742(1.269-2.393)$ & 0.001 & $1.610(1.168-2.219)$ & 0.004 \\
\hline Lymphonodus node metastasis (no vs. yes) & $2.030(1.415-2.912)$ & 0.000 & $2.335(1.646-3.312)$ & 0.000 \\
\hline Microvascular invasion (no vs. yes) & $1.292(0.885-1.886)$ & 0.185 & $1.521(0.919-2.515)$ & 0.103 \\
\hline $\mathrm{TNM}^{\mathrm{b}}$ (I+II vs. III+IVA) & $1.219(0.632-2.350)$ & 0.554 & $1.483(0.808-2.723)$ & 0.203 \\
\hline NLR (low vs. high) & $1.121(0.800-1.571)$ & 0.506 & $1.286(0.919-1.800)$ & 0.142 \\
\hline LMR (low vs. high) & $1.205(0.720-2.017)$ & 0.479 & $0.890(0.570-1.389)$ & 0.609 \\
\hline CA19-9, U/ml ( $\leq 89$ vs. $>89)$ & $1.650(1.216-2.238)$ & 0.001 & $1.302(0.747-2.270)$ & 0.352 \\
\hline \multicolumn{5}{|l|}{ SIS } \\
\hline I & 1 (Referent) & & 1 (Referent) & \\
\hline II & $1.262(0.871-1.829)$ & 0.219 & 1.307 (0.878- 1.947) & 0.187 \\
\hline III & $2.077(1.365-3.162)$ & 0.001 & $3.133(2.058-4.769)$ & 0.000 \\
\hline
\end{tabular}

Boldface type indicates significant values.

1 Analysis including LMR, CA19-9, and SIS.

Analyses were conducted using univariate analysis or multivariate Cox proportional hazards regression.

Abbreviations: AFP, alpha-fetoprotein; CA19-9, carbohydrate antigen 19-9; HBsAg, hepatitis B surface antigen; HCV, hepatitis C virus; LMR, lymphocyte-to-monocyte ratio; NLR, neutrophil-to-lymphocyte ratio; TTR, time to recurrence; OS, overall survival; TNM, tumor-node-metastasis; $\mathrm{CI}$, confidential interval; HR, hazard ratio; P, poor differentiation; M, moderated differentiation; W, well differentiation; SIS, combination of preoperative serum CA19-9 and LMR. I, CA19-9Low /LMR High; II, CA19-9 High/LMR High and CA19-9 Low/LMR Low; III, CA19-9 High/LMR Low.

a Tumor differentiation was determined according to the "British Society of Gastroenterology guidelines on the management of cholangiocarcinoma".

b TNM stage: American Joint Committee on Cancer 7th edition staging for intrahepatic cholangiocarcinoma.

Table 3. Multivariate analyses of prognostic factors in ICC (Cohort $1, n=322)$

\begin{tabular}{|c|c|c|c|c|}
\hline \multirow[t]{2}{*}{ Variable } & \multicolumn{2}{|l|}{ TTR } & \multicolumn{2}{|l|}{ OS } \\
\hline & HR (95\% CI) & $P$ & HR $(95 \% \mathrm{CI})$ & $P$ \\
\hline \multicolumn{5}{|l|}{ Multivariate analysis ${ }^{2}$} \\
\hline Tumor size, $\mathrm{cm}(\leq 5$ vs. $>5)$ & $1.034(0.751-1.422)$ & 0.838 & $1.019(0.736-1.409)$ & 0.912 \\
\hline Tumor number (single vs. multiple) & $1.761(1.281-2.421)$ & 0.000 & $1.701(1.235-2.345)$ & 0.001 \\
\hline Lymphonodus node metastasis (no vs. yes) & $1.972(1.375-2.829)$ & 0.000 & $2.177(1.533-3.091)$ & 0.000 \\
\hline Microvascular invasion (no vs. yes) & $1.582(0.971-2.577)$ & 0.066 & $1.627(0.985-2.689)$ & 0.058 \\
\hline TNM $^{\mathrm{b}}$ (I+II vs. III+IVA) & $1.289(0.671-2.478)$ & 0.446 & $2.065(1.498-2.848)$ & 0.000 \\
\hline NLR (low vs. high) & $1.113(0.793-1.562)$ & 0.536 & $1.504(1.108-2.043)$ & 0.009 \\
\hline LMR (low vs. high) & $0.758(0.557-1.032)$ & 0.079 & $0.636(0.461-0.878)$ & 0.006 \\
\hline CA19-9, U/ml ( $\leq 89$ vs. $>89)$ & $1.657(1.220-2.250)$ & 0.001 & $2.138(1.584-2.886)$ & 0.000 \\
\hline
\end{tabular}

Boldface type indicates significant values.

${ }^{2}$ Analysis including LMR and CA19-9 (omitting SIS)

Analyses were conducted using univariate analysis or multivariate Cox proportional hazards regression.

Abbreviations: CA19-9, carbohydrate antigen 19-9; LMR, lymphocyte-to-monocyte ratio; NLR, neutrophil-to-lymphocyte ratio; TTR, time to recurrence; OS, overall survival;

TNM, tumor-node-metastasis; CI, confidential interval; HR, hazard ratio.

a Tumor differentiation was determined according to the "British Society of Gastroenterology guidelines on the management of cholangiocarcinoma".

b TNM stage: American Joint Committee on Cancer 7th edition staging for intrahepatic cholangiocarcinoma. 


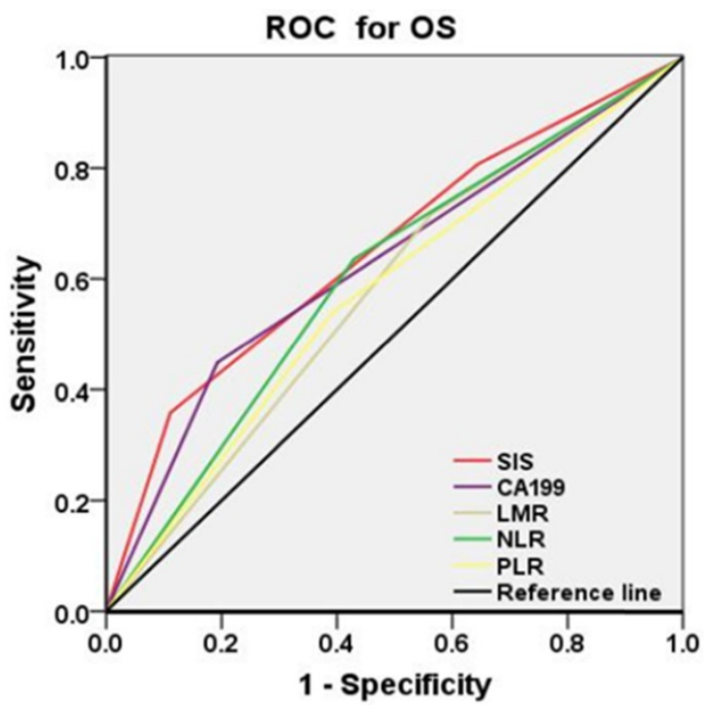

Figure 3. Predictive ability of the SIS was compared with other clinical parameters by ROC curves in the training cohort (Cohort $\mathbf{1}$, $n=322)$. The AUCs for SIS, CA19-9, LMR, NLR, and PLR were 0.652 (0.593-0.712, $P<0.001), 0.628$ (0.567-0.689, $P<0.001), 0.577$ (0.513-0.640, $P=0.019), 0.603$ (0.541-0.666, $P=0.002)$, and 0.574 (0.511-0.637, $P=0.024)$, respectively. Abbreviations: CA19-9, carbohydrate antigen 19-9; LMR, lymphocyte-to-monocyte ratio; NLR, neutrophil-to-lymphocyte ratio, PLR, platelet-lymphocyte ratio, SIS, systemic inflammation score. AUC, indicates area under the ROC curve.

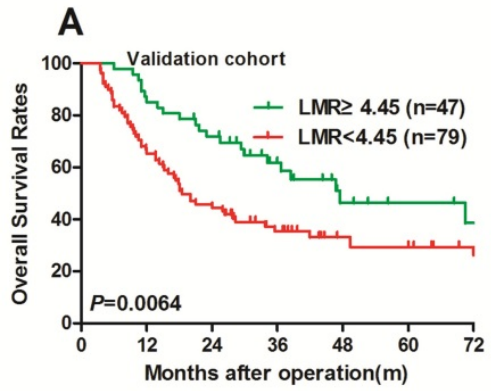

D

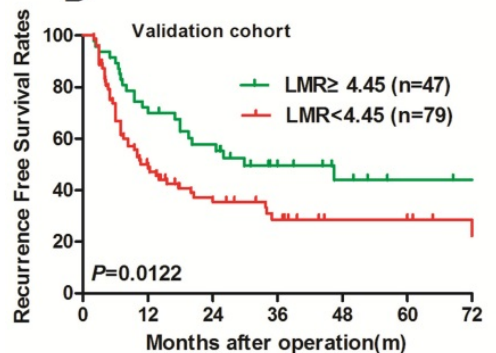

B

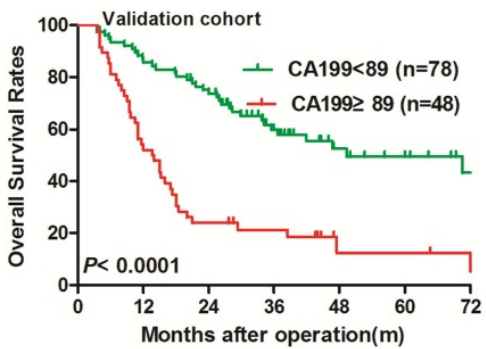

$E$

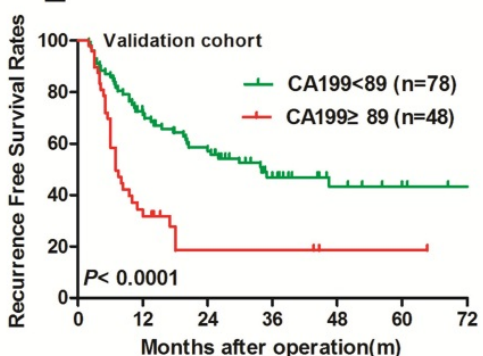

C

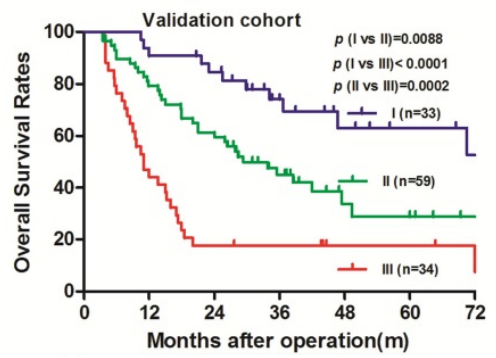

$\mathbf{F}$

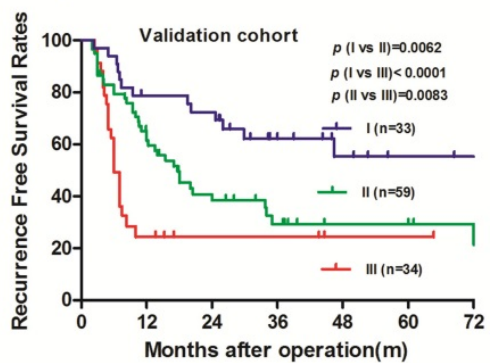

Figure 4. Prognostic values of preoperative LMR and serum CA19-9 using Kaplan-Meier analysis in the validation cohort (Cohort 2, $n=126)$. Kaplan-Meier analysis for overall survival according to (A) preoperative LMR, (B) preoperative serum CA19-9, (C) combination of preoperative LMR and serum CA19-9. Kaplan-Meier analysis for recurrence-free survival according to (D) preoperative LMR, (E) preoperative serum CA19-9, (F) combination of preoperative LMR and serum CA19-9. SIS, combination of preoperative LMR and serum CA19-9. I, CA19-9Low/LMRHigh; II, CA19-9 High/LMRHigh and CA19-9 Low/LMR Low; III, CA19-9 High/LMR Low.

\section{Discussion}

Growing evidence indicates a crucial role of cancer-related inflammation in cancer metastasis and progression. The hallmark of cancer-associated inflammation involves in the infiltration of inflammatory cells and the production of inflammatory mediators in tumor tissues, which participates in promotion of angiogenesis, extracellular matrix restructuring, and pre-metastatic niche formation [16]. Moreover, cancer-related inflammation generates alteration and polarization the tumor microenvironment that can increase propensity for tumor recurrence and metastasis [28, 29]. Serological inflammatory markers including CRP, NLR, LMR, and platelet-lymphocyte ratio (PLR) as well as tumor marker serum CA19-9, which reflect the host systemic inflammatory response have certain prognostic utilities in various cancers [24, 26, 30-32]. Therefore, the inflammatory mediators and inflammatory cells of tumor microenvironment may be reflected in the peripheral circulation.

Currently, there is no serum or bile tumor markers are specific for ICC but may be of diagnostic values. In the present study, our result identified 
serum CA19-9 as an independent predictor for RFS and OS. We chose the optimal cutoff value for serum CA19-9 was more than $89 \mathrm{U} / \mathrm{ml}$, which demonstrated the best discriminating power for distinguishing ICC patient clinical outcome in our large cohort. Furthermore, high CA19-9 was significantly correlated with high Child-Pugh score, large tumor size, lymphonodus node metastasis, and high TNM stage. This finding parallels previous studies have identified serum CA19-9 greater than $100 \mathrm{U} / \mathrm{ml}$ was significantly correlated with inferior RFS after hepatic resection [33]. Therefore, elevated serum CA19-9 may facilitate the selection of the ICC patients with an aggressive tumor biology. This group of patients should be monitor and further postoperative adjuvant treatment be carried out.

The development of tumor metastasis comprises a complex cascade steps that involves the multiple intercellular interaction between the tumor cells and the host-derived stromal cells that includes factors that facilitate angiogenesis and pre-metastatic niche formation. Several studies have demonstrated the prognostic utilities of NLR, PLR, and CA19-9 in ICC patients after surgery [24, 26, 34]. Although previous study examining the LMR in ICC, had been limited both in size and scope to select populations within locally advanced or metastatic ICC [17]. In the present study, ROC and AUC analysis identified serum CA19-9 and LMR as a predictor for OS. Our results revealed that elevated serum CA19-9 and low LMR were independent and worse predictors of OS and TTR in two independent cohorts. Furthermore, multivariate analysis identified preoperative serum CA19-9 and LMR were also independent predictor for OS and TTR, after excluding SIS. Consistent with the recent reports, evaluating the convenient clinical application of LMR as an easily measurable inflammatory biomarker in a variety of solid tumors, such as HCC, lung, and colon cancer [31, 35-37].

In the present study, we constructed a novel prognostic score named SIS basing on serum CA19-9 and LMR, which were both confirmed to be correlated with ICC patients' outcome. Furthermore, we also assessed the prognostic utility of combined serum CA19-9 and LMR in patients with ICC by direct comparisons of prognosis among three subgroups (I, CA19-9Low/LMR High; II, CA19-9 High/LMR High and CA19-9 Low/LMR Low; III, CA19-9 High/LMR Low) in 322 ICC patients. Our results demonstrated that ICC patients who had both elevated serum CA19-9 and low LMR were more prone to early recurrence and suffered poor survival rates after hepatectomy. Conversely, the ICC patients who had both low serum CA19-9 and high LMR had the best prognosis. Multivariate analysis indicated that high SIS score was a significant independent inferior predictor for OS and TTR, together with tumor number and tumor-node metastasis stage. Consistent with our previous findings demonstrated that high systemic immune-inflammation index was a powerful prognostic indicator of poor outcome in HCC patients [38]. Moreover, elevated SIS score was significantly associated with aggressive tumor biological phenotypes such as high TNM stage, large tumor size, multiple tumors, lymphonodus node metastasis, and high AFP level, as well as HBsAg positive. Therefore, the predictive significance of the SIS score in those subgroups may facilitate clinicians identifying patients with high risk of recurrence and enable targeted rational adjuvant therapy after hepatic resection.

As an integrated indicator basing on serum tumor marker CA19-9 and LMR, the potential mechanisms behind the prognostic utility of SIS might be elucidated by the function of the serum CA19-9, lymphocytes and monocytes. Here, we confirmed the elevated CA19-9 was powerful prognostic indicator of poor outcome in patients with ICC, and was also in keeping with those previous findings [22, 32]. Actually, serum CA19-9 is not specific for ICC but may be of diagnostic utility. Serum tumor markers CA19-9 and CEA are significant overlapped with other benign diseases. In addition, acute cholangitis or bile duct obstruction may affect serum CA19-9 level. Previous literature had shown that lymphocytes can strengthen cancer immune-surveillance to suppress tumor cell proliferation, invasion and metastasis [39]. Lymphopenia has been associated with worse outcomes in cancer patients [40,41]. Recent studies demonstrates that serum monocytes can be recruited in tumor microenvironment and differentiate into tumor-associated macrophages (TAMs), which participate incancer angiogenesis, immunosuppression, and metastasis [42, 43]. In line with the literatures, our earlier results confirmed that myeloid-derived suppressor cells are a subset of circulating leucocytes known to have immunosuppressive activity in HCC [31, 44]. Therefore, a low circulating lymphocyte amounts indicate impaired immunity, which may facilitate tumorigenesis and subsequent tumor growth. An increased circulating serum levels of monocytes may indicate an elevated levels of TAMs as a marker of high tumor burden.

This is the first study, to our knowledge, to describe the use of systemic inflammation score (SIS) based on preoperative serum CA19-9 and LMR, which are routine laboratory parameters that reflect the systemic inflammatory response as a biomarker for ICC patients undergoing curative intent resection. 
Meanwhile, the measure of SIS is based on standard laboratory measurements of total monocyte, and lymphocyte counts as well as serum tumor marker CA19-9, which are routinely performed in clinical practice. Thus, our results indicated that the SIS may be a powerful prognostic biomarker for ICC recurrence and treatment response surveillance, which may allow early therapeutic intervention according to characteristics of individual tumors.

Previously, we have observed that decreased PNI, another inflammatory marker, predicted poor postoperative prognosis of advance ICC patients, especially in those with metastatic ICC [17]. However, our current results indicated that PNI was not a promising independent prognostic factor. Our results were consistent with the previous studies [45]. The exclusion criteria of ICC patients enrolled in our cohorts included intrahepatic metastasis of extrahepatic cholangiocarcinoma, which may explain why preoperative PNI was not an independent prognostic factor in current study. In addition, we did not evaluate mGPS in our analyses, because serum CRP was not routinely performed at our institution. The prognostic utility of mGPS in combination with SIS should be evaluated in the further studies.

The current study has several limitations. First, it was a retrospective analysis. There may have been a selection bias regarding the diagnosis and treatment of patients with ICC in the study. Second, most ICC patients $(n=123$ [38.2\%] in primary cohort) in China were seropositive for hepatitis B surface antigen, whereas one patient $(n=1[0.31 \%]$ in primary cohort) had anti HCV positivity, which differs greatly from the patient population in previous studies in United States, Europe, and Japan. Because the key detrimental factors in carcinogenesis of ICC are primary sclerosing cholangitis and $\mathrm{HCV}$ infection, especially in western countries. Therefore, the prognostic significance of the SIS needs to be validated in patients with ICC from other populations in the future. Nonetheless, more prospective studies should be conducted to further validate the predicting probability of SIS.

\section{Conclusions}

In conclusion, we created an easily obtained systemic inflammatory biomarker named SIS basing on preoperative serum CA19-9 and LMR. Our results have demonstrated the SIS qualifies as a novel, independent prognostic predictor of patients with ICC after hepatectomy in two independent cohorts. Our results confirmed that the elevated SIS was associated with early recurrence and poor prognosis in patients with ICC after curative resection. The preoperative serum CA19-9 and the blood tests be routinely performed in the clinical practice, which make the SIS a promising tool for assessing ICC prognosis and personalizing management in future clinical practice.

\section{Abbreviations}

AFP, alpha-fetoprotein; ICC, Intrahepatic Cholangiocarcinoma; HR, hazard ratio; OS, overall survival; Systemic inflammation score, SIS.

\section{Supplementary Material}

Supplementary figures and tables.

http://www.jcancer.org/v10p0494s1.pdf

\section{Acknowledgements}

This study was supported grants from the National Natural Science Funds of China (No.81702893; No.81372650), the Shanghai Public Health Clinical Center (SPHCC-2018-12), and the National Key research and development program (2016YFC090240; 2017ZX10203204).

\section{Author contributions}

Concept and design: Yong Zhang, Shi-Ming Shi, Hua Yang, Jian Zhou, Qing Chen.

Provision of study materials or patients: Yong Zhang, Liu-Xiao Yang, Zheng Wang, Xue-Dong Li, Ying-Hong Shi, Ya Cao, Jian Zhou.

Collection and/or assembly of data: Shi-Ming Shi, Liu-Xiao Yang, Zheng Wang, Xue-Dong Li, Dan Yin, Qing Chen.

Data analysis and interpretation: Yong Zhang, Shi-Ming Shi, Liu-Xiao Yang, Hua Yang, Zhi Dai, Jian Zhou, Qing Chen.

Manuscript writing: Yong Zhang, Shi-Ming Shi, Liu-Xiao Yang, Hua Yang, Jian Zhou, Qing Chen.

Final approval of manuscript: Qing Chen, Jian Zhou.

\section{Competing Interests}

The authors have declared that no competing interest exists.

\section{References}

1. Kaczynski J, Hansson G, Wallerstedt S. Incidence, etiologic aspects and clinicopathologic features in intrahepatic cholangiocellular carcinoma--a study of 51 cases from a low-endemicity area. Acta Oncol 1998; 37: 77-83.

2. Liver Cancer Study Group of J. Primary liver cancer in Japan. Clinicopathologic features and results of surgical treatment. Ann Surg 1990; 211: 277-287.

3. Altekruse SF, Petrick JL, Rolin AI, et al. Geographic variation of intrahepatic cholangiocarcinoma, extrahepatic cholangiocarcinoma, and hepatocellular carcinoma in the United States. PLoS One 2015; 10: e0120574.

4. Patel T. Increasing incidence and mortality of primary intrahepatic cholangiocarcinoma in the United States. Hepatology 2001; 33: 1353-1357.

5. Farges O, Fuks D, Boleslawski E, et al. Influence of surgical margins on outcome in patients with intrahepatic cholangiocarcinoma: a multicenter study by the AFC-IHCC-2009 study group. Ann Surg 2011; 254: 824-829; discussion 830 . 
6. Tan JC, Coburn NG, Baxter NN, et al. Surgical management of intrahepatic cholangiocarcinoma--a population-based study. Ann Surg Oncol 2008; 15: 600-608.

7. Bridgewater J, Galle PR, Khan SA, et al. Guidelines for the diagnosis and management of intrahepatic cholangiocarcinoma. J Hepatol 2014; 60: 1268-1289.

8. Zaydfudim VM, Rosen CB, Nagorney DM. Hilar cholangiocarcinoma. Surg Oncol Clin N Am 2014; 23: 247-263.

9. de Jong $\mathrm{MC}$, Nathan $\mathrm{H}$, Sotiropoulos $\mathrm{GC}$, et al. Intrahepatic cholangiocarcinoma: an international multi-institutional analysis of prognostic factors and lymph node assessment. J Clin Oncol 2011; 29: 3140-3145.

10. DeNardo DG, Johansson M, Coussens LM. Immune cells as mediators of solid tumor metastasis. Cancer Metastasis Rev 2008; 27: 11-18.

11. Mantovani A, Allavena P, Sica A, et al. Cancer-related inflammation. Nature 2008; 454: 436-444.

12. El-Serag HB, Engels EA, Landgren $\mathrm{O}$, et al. Risk of hepatobiliary and pancreatic cancers after hepatitis $C$ virus infection: A population-based study of U.S. veterans. Hepatology 2009; 49: 116-123.

13. Sempoux C, Jibara G, Ward SC, et al. Intrahepatic cholangiocarcinoma: new insights in pathology. Semin Liver Dis 2011; 31: 49-60.

14. Cai H, Kong WT, Chen CB, et al. Cholelithiasis and the risk of intrahepatic cholangiocarcinoma: a meta-analysis of observational studies. BMC Cancer 2015; 15: 831.

15. Hanahan D, Weinberg RA. Hallmarks of cancer: the next generation. Cell 2011; 144: 646-674.

16. Mantovani A. Cancer: Inflaming metastasis. Nature 2009; 457: 36-37.

17. Zhang $\mathrm{C}$, Wang $\mathrm{H}$, Ning $\mathrm{Z}$, et al. Prognostic nutritional index serves as a predictive marker of survival and associates with systemic inflammatory response in metastatic intrahepatic cholangiocarcinoma. Onco Targets Ther 2016; 9: 6417-6423.

18. Song W, Wang K, Zhang RJ, et al. Prognostic value of the lymphocyte monocyte ratio in patients with colorectal cancer: A meta-analysis. Medicine (Baltimore) 2016; 95: e5540.

19. Yoshida T, Kinoshita H, Yoshida K, et al. Prognostic impact of perioperative lymphocyte-monocyte ratio in patients with bladder cancer undergoing radical cystectomy. Tumour Biol 2016; 37: 10067-10074.

20. Yang T, Zhu J, Zhao L, et al. Lymphocyte to monocyte ratio and neutrophil to lymphocyte ratio are superior inflammation-based predictors of recurrence in patients with hepatocellular carcinoma after hepatic resection. J Surg Oncol 2017; 115: 718-728.

21. Halazun KJ, Hardy MA, Rana AA, et al. Negative impact of neutrophil-lymphocyte ratio on outcome after liver transplantation for hepatocellular carcinoma. Ann Surg 2009; 250: 141-151.

22. Yoh T, Seo S, Hatano E, et al. A Novel Biomarker-Based Preoperative Prognostic Grading System for Predicting Survival After Surgery for Intrahepatic Cholangiocarcinoma. Ann Surg Oncol 2017; 24: 1351-1357.

23. Proctor MJ, Morrison DS, Talwar D, et al. An inflammation-based prognostic score (mGPS) predicts cancer survival independent of tumour site: a Glasgow Inflammation Outcome Study. Br J Cancer 2011; 104: 726-734.

24. Chen $\mathrm{Q}$, Yang LX, Li XD, et al. The elevated preoperative neutrophil-to-lymphocyte ratio predicts poor prognosis in intrahepatic cholangiocarcinoma patients undergoing hepatectomy. Tumour Biol 2015; 36: 5283-5289.

25. Gu FM, Gao Q, Shi GM, et al. Intratumoral IL-17(+) cells and neutrophils show strong prognostic significance in intrahepatic cholangiocarcinoma. Ann Surg Oncol 2012; 19: 2506-2514

26. Chen Q, Dai Z, Yin D, et al. Negative impact of preoperative platelet-lymphocyte ratio on outcome after hepatic resection for intrahepatic cholangiocarcinoma. Medicine (Baltimore) 2015; 94: e574.

27. Gao Q, Zhao YJ, Wang XY, et al. Activating mutations in PTPN3 promote cholangiocarcinoma cell proliferation and migration and are associated with tumor recurrence in patients. Gastroenterology 2014; 146: 1397-1407.

28. Chechlinska M, Kowalewska M, Nowak R. Systemic inflammation as a confounding factor in cancer biomarker discovery and validation. Nat Rev Cancer 2010; 10: 2-3

29. Brandau S, Dumitru CA, Lang S. Protumor and antitumor functions of neutrophil granulocytes. Semin Immunopathol 2013; 35: 163-176.

30. Hashimoto K, Ikeda Y, Korenaga D, et al. The impact of preoperative serum C-reactive protein on the prognosis of patients with hepatocellular carcinoma. Cancer 2005; 103: 1856-1864.

31. Chan JC, Chan DL, Diakos CI, et al. The Lymphocyte-to-Monocyte Ratio is a Superior Predictor of Overall Survival in Comparison to Established Biomarkers of Resectable Colorectal Cancer. Ann Surg 2017; 265: 539-546.

32. Yamashita S, Passot G, Aloia TA, et al. Prognostic value of carbohydrate antigen 19-9 in patients undergoing resection of biliary tract cancer. Br J Surg 2017; 104: 267-277.

33. Tamandl D, Herberger B, Gruenberger B, et al. Influence of hepatic resection margin on recurrence and survival in intrahepatic cholangiocarcinoma. Ann Surg Oncol 2008; 15: 2787-2794.

34. Li FH, Chen XQ, Luo HY, et al. [Prognosis of 84 intrahepatic cholangiocarcinoma patients]. Ai Zheng 2009; 28: 528-532.

35. Stotz M, Pichler M, Absenger G, et al. The preoperative lymphocyte to monocyte ratio predicts clinical outcome in patients with stage III colon cancer. Br J Cancer 2014; 110: 435-440.
36. Lin ZX, Ruan DY, Li Y, et al. Lymphocyte-to-monocyte ratio predicts survival of patients with hepatocellular carcinoma after curative resection. World J Gastroenterol 2015; 21: 10898-10906.

37. Go SI, Kim RB, Song $\mathrm{HN}$, et al. Prognostic significance of the lymphocyte-to-monocyte ratio in patients with small cell lung cancer. Med Oncol 2014; 31: 323.

38. $\mathrm{Hu} \mathrm{B}$, Yang $\mathrm{XR}, \mathrm{Xu} \mathrm{Y}$, et al. Systemic immune-inflammation index predicts prognosis of patients after curative resection for hepatocellular carcinoma. Clin Cancer Res 2014; 20: 6212-6222.

39. Dunn GP, Old LJ, Schreiber RD. The immunobiology of cancer immunosurveillance and immunoediting. Immunity 2004; 21: 137-148.

40. Fogar P, Sperti C, Basso D, et al. Decreased total lymphocyte counts in pancreatic cancer: an index of adverse outcome. Pancreas 2006; 32: 22-28.

41. Ray-Coquard I, Cropet C, Van Glabbeke M, et al. Lymphopenia as a prognostic factor for overall survival in advanced carcinomas, sarcomas, and lymphomas. Cancer Res 2009; 69: 5383-5391.

42. Qian BZ, Pollard JW. Macrophage diversity enhances tumor progression and metastasis. Cell 2010; 141: 39-51.

43. Zhou SL, Zhou ZJ, Hu ZQ, et al. Tumor-Associated Neutrophils Recruit Macrophages and T-Regulatory Cells to Promote Progression of Hepatocellular Carcinoma and Resistance to Sorafenib. Gastroenterology 2016; 150: 1646-1658 e1617.

44. Yang $X$, Lin $Y$, Shi $Y$, et al. FAP Promotes Immunosuppression by Cancer-Associated Fibroblasts in the Tumor Microenvironment via STAT3-CCL2 Signaling. Cancer Res 2016; 76: 4124-4135.

45. Okuno M, Ebata T, Yokoyama Y, et al. Appraisal of inflammation-based prognostic scores in patients with unresectable perihilar cholangiocarcinoma. J Hepatobiliary Pancreat Sci 2016; 23: 636-642. 